TRANSACTIONS OF THE

AMERICAN MATHEMATICAL SOCIETY

Volume 190,1974

\title{
KOEBE SEQUENCES OF ARCS AND NORMAL MEROMORPHIC FUNCTIONS
}

BY

\section{STEPHEN DRAGOSH}

ABSTRACT. Let $f$ be a normal meromorphic function in the unit disk. An estimate for the growth of the modulus of $f$ on a Koebe sequence of arcs is obtained; the estimate is in terms of the order of normality of $f$. An immediate consequence of the estimate is the following theorem due to F. Bagemihl and W. Seidel: A nonconstant normal meromorphic function has no Koebe values. Another consequence is that each level set of a nonconstant normal meromorphic function cannot contain a Koebe sequence of arcs provided the order of normality of $f$ is less than a certain positive constant $C^{*}$.

1. Introduction. $A$ meromorphic function $f$ is normal in the unit disk $D$ : $|z|<1$ if and only if the family $\{f(S(z))\}$ is normal in $D$ in the sense of Montel, where $S$ is any conformal map of $D$ onto itself. K. Noshiro [10, Theorem 1], and subsequently $O$. Lehto and K. I. Virtanen [6, Theorem 3], employed the spherical derivative

$$
f^{*}(z)=\frac{\left|f^{\prime}(z)\right|}{1+|f(z)|^{2}}
$$

to obtain the following characterization of normal meromorphic functions: $f$ is normal in $D$ if and only if

$$
C=C_{f}=\sup _{z \in D}\left(1-|z|^{2}\right) f^{*}(z)<+\infty ;
$$

$C$ is called the order of normality of $f$. Using integration, we deduce from (1.1) that an arc $\gamma$ in $D$ with non-Euclidean length $\rho$ is mapped by $f$ onto an arc with spherical length not exceeding $C \rho$. Thus, $f$ is "more normal" than $g$ when $C_{f}<C_{8^{*}}$

Throughout this paper we make use of Pick's (differential) form of Schwarz's lemma. If $\phi$ is an analytic mapping of $D$ into itself, then

$$
\left(1-|z|^{2}\right)\left|\phi^{\prime}(z)\right| \leq 1-|\phi(z)|^{2} ;
$$

thus $C_{\phi} \leq 1$, and $C_{f(\phi)} \leq C_{f}$ for any function $f$ meromorphic in $D$. Equality holds in all cases if $\phi$ is a conformal map of $D$ onto itself.

Receired by the editors J anuary 5, 1973.

AMS (MOS) subject classifications (1970). Primary 30A74; Secondary 30A72.

Key words and phrases. Normal meromorphic function, order of normality, Koebe lemma, Koebe sequence of arcs, level set, boundary behavior. 
If $f$ is defined in $D$ and $\zeta$ is a point of the unit circle $\Gamma:|z|=1$, we set

$$
|f(\zeta)|=\underset{z \rightarrow \zeta}{\lim \sup }|f(z)| \text {. }
$$

In the terminology of cluster sets, $|f(\zeta)|$ is the radius of the smallest closed disk such that the disk has center zero and contains all the cluster values (boundary values) of $f$ at $\zeta$. If $|f(\zeta)| \leq M<+\infty$ for all points $\zeta$ in a subarc $\gamma$ of $\Gamma$, then $f$ is said to be bounded (by $M$ ) on $\gamma$. By combining the classical two-constants estimate for $|f(z)|$ and the estimate (1.1) for $\left|f^{\prime}(z)\right|$, Lehto and Virtanen obtained a "two-constants theorem" for normal meromorphic functions bounded on a subarc $\gamma$. Several related results and examples are discussed in $\$ 2$.

In $\$ 3$ we prove the central result of this paper: An estimate for the growth of the modulus of a normal meromorphic function on a Koebe sequence of arcs. Following F. Bagemihl and W. Seidel [1], a sequence of disjoint Jordan arcs $\left\{\gamma_{n}\right\}$ in $D$ is called a Koebe sequence of arcs relative to an open subarc $\gamma$ of $\Gamma$ provided (i) each neighborhood of $\gamma$ contains all but finitely many of the arcs $\gamma_{n}$, and (ii) every open sector $\Delta$ of $D$ subtending a subarc whose closure lies in $\gamma$ has the property that all but finitely many of the arcs $\gamma_{n}$ contain at least one Jordan subarc lying entirely in $\Delta$ except for its two endpoints which lie on distinct sides of $\Delta$. If $f$ is defined in $D$ and $\left\{\gamma_{n}\right\}$ is a Koebe sequence of arcs, we set

$$
m_{n}=m_{n}\left(\gamma_{n} ; f\right)=\inf _{z \in \gamma_{n}}|f(z)| \text { and } M_{n}=M_{n}\left(\gamma_{n} ; f\right)=\sup _{z \in \gamma_{n}}|f(z)| .
$$

If $\lim M_{n}=0$, zero is called a Koebe value of $f$. More generally, the complex number $c$ is a Koebe value of $f$ provided zero is a Koebe value of $f(z)-c$ or $1 / f$, according as $c$ is finite or $c=\infty$. Koebe's lemma states that a nonconstant bounded analytic function has no Koebe values.

Some estimates that we obtain for the growth of $|f|$ are the following (Theorem 1): If $f$ is a meromorphic function in $D$ with order of normality $C>0$ and $\left\{\gamma_{n}\right\}$ is a Koebe sequence of arcs relative to an open subarc $\gamma$ of $\Gamma$, then

$$
\lim \inf M_{n} \geq \frac{1+\left(1+C^{2}\right)^{1 / 2}}{C} \exp \left[-\left(1+C^{2}\right)^{1 / 2}\right]
$$

or

$$
\lim \inf M_{n} \geq \sup _{\zeta \in \gamma}|f(\zeta)| \text {, }
$$

according as $f$ is unbounded or bounded on $\gamma$. By combining (1.3) and (1.4) (or (1.3) and Koebe's lemma), we reestablish a result of Bagemihl and Seidel [1, Theorem 1]: A nonconstant normal meromorphic function cannot have zero (and hence any extended complex number) as a Koebe value.

Since $f$ and $1 / f$ have equal spherical derivatives, $C_{f}=C_{1 / f}$ We infer from (1.3) that if $1 / f$ is not bounded on $\gamma$, then 


$$
\lim \sup m_{n} \leq \frac{C}{1+\left(1+C^{2}\right)^{1 / 2}} \exp \left[\left(1+C^{2}\right)^{1 / 2}\right] .
$$

In our investigations, a fundamental role is played by the number $C=C^{*}$ for which the right-hand sides of (1.3) and (1.5) are equal (and hence equal to one); $C^{*} \approx 2 / 3$. In particular, if both $f$ and $1 / f$ are unbounded on $\gamma$ and $C_{f}<C^{*}$, then

$$
\lim \sup m_{n}<\lim \inf M_{n}
$$

In $\$ 4$ we consider the family $\mathcal{L}$. A nonconstant meromorphic function $f \in \mathcal{L}$ if and only if every level set of $f$ does not contain a Koebe sequence of arcs. A lemma of G. R. MacLane $[7$, p. 11] states that each nonconstant bounded analytic

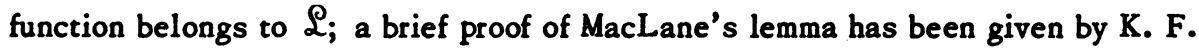
Barth and W. J. Schneider [2]. More generally, MacLane [7, Theorems 1 and 17] proved that each nonconstant normal analytic function belongs to $\mathcal{L}$. We prove the following results: (Lemma 2) If the nonconstant normal meromorphic function $f$ has a level set $L_{R}$,

$$
L_{R}=\{z:|f(z)|=R\},
$$

such that $L_{R}$ contains a Koebe sequence of arcs relative to an open subarc $\gamma$ of $\Gamma$, then

$$
\frac{1+\left(1+C^{2}\right)^{1 / 2}}{C} \exp \left[-\left(1+C^{2}\right)^{1 / 2}\right] \leq R \leq \frac{C}{1+\left(1+C^{2}\right)^{1 / 2}} \exp \left[\left(1+C^{2}\right)^{1 / 2}\right], \quad C^{*} \leq C=C_{f}
$$

and each point of $\gamma$ is a limit point of zeros and poles of $f$; thus (Theorem 2) if either $f$ is a nonconstant normal analytic function in $D$ or $C=C$, satisfies $0<C<C^{*}$, then $f \in \mathfrak{L}$. Moreover, (Theorem 3)

$$
C^{*}=\inf \left\{C_{f}: f \notin \mathscr{L}, C_{f}>0\right\} \text {. }
$$

Whether or not there exists a normal meromorphic function $f \notin \mathscr{L}$ such that $C^{*}=C$, is an open question.

Finally, in $\$ 5$ we discuss the boundary behavior of normal meromorphic functions $f$ for which $|f|$ has a Koebe value.

2. Boundary estimates for normal functions. Let $G_{a}$ be a lens (crescent) bounded by the unit circle $\Gamma:|z|=1$ and a circular arc in $D$ that intersects $\Gamma$ at an angle $a, 0<a<\pi$. Excluding the vertices of $G_{a}$, the boundary of $G_{a}$ consists of an open subarc $\Gamma_{\beta}$ of $\Gamma$ such that $\Gamma-\Gamma_{\beta}$ is viewed under the angle $\beta(0<\beta<\pi)$ from $\Gamma_{\beta}$ and an open circular arc $\Gamma_{\delta}$ in $D$ such that $\Gamma-\Gamma_{\beta}$ is viewed under the angle $\delta(\beta<\delta<\pi+\beta)$ from $\Gamma_{\delta}$. Note that $a=\delta-\beta$. The harmonic measure $\omega$ of $\Gamma_{\beta}$ with respect to $G_{\alpha}$ is given by

$$
\omega\left(z, \Gamma_{\beta}, G_{\alpha}\right)=\alpha^{-1}(\delta-\theta) \quad(\beta<\theta<\delta),
$$


where $\theta$ is the angle under which $\Gamma-\Gamma_{\beta}$ is viewed from $z$. In the theorem to follow, we make use of the expression

$$
\lambda(z)=\frac{1}{1-|z|^{2}} / \frac{\partial \omega}{\partial n} \quad\left(z \in \Gamma_{\delta}\right),
$$

where $\partial \omega / \partial n$ is the normal derivative of $\omega$ at $z \in \Gamma_{\delta}$. Using (1.2), we find that $\lambda(z)\left(z \in G_{\alpha}\right)$ is invariant under a conformal map $z=\phi\left(z^{\prime}\right)$ of $D$ onto itself.

Therefore, we can assume that $\Gamma_{\delta}$ passes through $z=0$ and that the positive real axis is the inner normal to $\Gamma_{\delta}$ at $z=0$. Some elementary calculations yield

$$
\lambda_{a}=\left.\lambda(z)\right|_{z \in \Gamma_{\delta}}=\frac{a}{2 \sin \alpha} \text {. }
$$

In this setting, Lehto and Virtanen's "two-constants theorem" assumes the following form.

Theorem A. Let $f$ be meromorpbic in $D$ with order of normality $C(0<C<$ $+\infty)$, and suppose

$$
\sup _{z \in G_{a}}|f(z)| \geq \mu \text {. }
$$

If $f$ is bounded by $M$ on the open arc $\Gamma_{\beta}$, then

$$
M \geq \mu \exp \left[-C \lambda_{a}(\mu+1 / \mu)\right] \text {. }
$$

If

$$
\mu_{a}=\frac{1+\left(1+4 C^{2} \lambda_{a}^{2}\right)^{1 / 2}}{2 C \lambda_{a}},
$$

then the best estimates in (2.1) are obtained by setting

and

$$
\boldsymbol{\mu}=\sup _{\boldsymbol{z} \in G_{\alpha}}|f(z)|, \quad \text { if } \sup _{\boldsymbol{z} \in G_{\alpha}}|f(z)|<\mu_{\alpha},
$$

$$
\mu=\mu_{a}, \quad \text { if } \sup _{z \in G_{a}}|f(z)| \geq \mu_{\alpha} .
$$

In their version of Theorem $A$, Lehto and Virtanen assumed that $f$ is bounded by $M$ on the closure of the arc $\Gamma_{\beta}$; our weaker form is easily verified by an obvious limiting argument using closed subarcs of $\Gamma_{\beta}$.

We briefly indicate once again how the best estimates are obtained. As a function of $\mu \geq 0$,

$$
\mu \exp \left[-C \lambda_{\alpha}(\mu+1 / \mu)\right]
$$

has its maximum at $\mu=\mu_{a}$. Therefore, if

$$
\sup _{z \in G_{a}}|f(z)| \geq \mu_{a}
$$


we can choose $\mu=\mu_{a}$ in (2.1) and hence maximize its right-hand side. If

then

$$
\sup _{z \in G_{\alpha}}|f(z)|<\mu_{a}
$$

$$
\mu=\sup _{\boldsymbol{z} \in G_{\alpha}}|f(z)|
$$

maximizes the right-hand side of (2.1).

If $f$ is bounded by $M$ on $\Gamma$ with the (possible) exception of one point, then Theorem A, as stated, does not apply. However, by using a limiting process (as $a \rightarrow 0$ ). Lehto and Virtanen deduced the following estimate of the PharagménLindelöf type.

Theorem B. Let $f$ be meromorphic in $D$ with order of normality $C(0<C<+\infty)$, and let $f$ be bounded by $M$ on $\Gamma-\{\zeta\}, \zeta \in \Gamma$. Then

$$
M \geq \frac{1+\left(1+C^{2}\right)^{1 / 2}}{C} \exp \left[-\left(1+C^{2}\right)^{1 / 2}\right]
$$

unless $f$ is bounded in $D$, in whicb case $M \geq C$.

As noted in $[6, \mathrm{p} .62]$, the estimate $(2.2)$ is sharp. The function

$$
f(z)=M \exp (b(1+z) /(1-z)) \quad(M>0, b>0)
$$

is bounded by $M$ on $\Gamma-\{1\}$ and equality holds in (2.2) for $C=C_{f}$.

We now investigate the sharpness of the estimate (2.2) in case $f$ is bounded by $M$ on all of $\Gamma$ and unbounded in $D$. To simplify notation, we henceforth let $C=C_{M}^{*}$ be the solution of

$$
M=\frac{1+\left(1+C^{2}\right)^{1 / 2}}{C} \exp \left[-\left(1+C^{2}\right)^{1 / 2}\right]
$$

As in the introduction, we set $C^{*}=C_{1}^{*}$. Using this notation, (2.2) is equivalent to the inequality $C_{M}^{*} \leq C$.

Example 1. Let $M>0$, and set $C_{n}=C_{f_{n^{\prime}}}$ where $f_{n}(z)=M z^{-n}(n=2,3, \ldots)$. Then $C_{M}^{*} \leq C_{n}=C_{M}^{*}+o(1)$.

Proof. The inequality $C_{M}^{*} \leq C_{n}$ follows from Theorem B. Let

Then

$$
\Phi_{n}(z) \doteq\left(1-|z|^{2}\right) f_{n}^{*}(z)=\left(1-|z|^{2}\right) \frac{M n|z|^{n-1}}{M^{2}+|z|^{2 n}} \text {. }
$$

$$
C_{n}=\max _{0 \leq r \leq 1} \Phi_{n}(r)
$$

We have

$$
\Phi_{n}^{\prime}(r)=\frac{M n r^{n-2}}{\left(M^{2}+r^{2 n}\right)^{2}}\left[(n-1) r^{2 n+2}-(n+1) r^{2 n}-M^{2}(n+1) r^{2}+M^{2}(n-1)\right] .
$$


Now, for all values of $n$,

$$
\Phi_{n}^{\prime}(n /(n+1))<0
$$

and, for all sufficiently large values of $n$,

$$
\Phi_{n}^{\prime}\left(\left(n-M^{-2}\right) /(n+1)\right)>0 .
$$

To verify (2.6), we note that as $n \rightarrow+\infty$ in (2.6), we obtain the valid inequality

$$
2>\left(4+2 M^{-2}\right) \exp \left[-2\left(1+M^{-2}\right)\right] \text {. }
$$

From (2.5) and (2.6), it follows that

$$
\left.\Phi_{n}^{\prime}\left(\left(n-t_{n}\right)\right)^{\prime}(n+1)\right)=0
$$

for some $t_{n}$ satisfying $0<t_{n}<M^{-2}$.

Because $\Phi_{n}^{\prime}$ has exactly one zero for $0<r<1$,

$$
C_{n}=\Phi_{n}\left(\left(n-t_{n}\right) /(n+1)\right) \text {. }
$$

If $t^{\prime}$ is a limit point of the sequence $\left\{t_{n}\right\}$ and we formally compute the limit as $n \rightarrow+\infty$ in (2.7), we find that $t^{\prime}$ satisfies

$$
t+2=M^{2} t e^{2(1+t)} \text {. }
$$

However, (2.9) has exactly one solution for $t>0$ and thus $t^{\prime}=\lim t_{n}$. Moreover, since $t^{\prime}$ satisfies (2.9), $t^{\prime}$ maximizes the function

$$
\psi(t)=\frac{2 M(t+1) e^{1+t}}{1+M^{2} e^{2(1+t)}}
$$

for which $\psi^{\prime}(t)=0$ reduces to (2.9). If we set $t^{\prime}=\left(1+C^{2}\right)^{1 / 2}-1$, then some elementary computations show that

$$
\psi\left(t^{\prime}\right)=\psi\left(\left(1+C^{2}\right)^{1 / 2}-1\right)=C
$$

and that $C$ satisfies (2.4). Thus $C=C_{M}^{*}$ and we find

$$
\lim C_{n}=\lim \Phi_{n}\left(\left(n-t_{n}\right) /(n+1)\right)=\psi\left(t^{\prime}\right)=C=C_{M^{\bullet}}^{*}
$$

Example 1 can be verified by another method that points out a connection between the functions $M z^{-n}$ and the function in (2.3); this method is based on the following lemma.

Lemma 1. Let $\left\{g_{n}\right\}$ be a sequence of normal meromorphic functions that converges uniformly to $g$ on compact subsets of $D$. Let $C_{n}=C_{8_{n}}$. Then

$$
C_{g} \leq \lim \text { inf } C_{n^{*}}
$$

If there exists an $r(0 \leq r<1)$ such that 


$$
C_{n}=\left(1-\left|z_{n}\right|^{2}\right) g_{n}^{*}\left(z_{n}\right), \quad\left|z_{n}\right| \leq r
$$

then $C_{8}=\lim C_{n^{\circ}}$

Proof. Let $\left\{g_{n_{k}}\right\}$ be a subsequence such that $\lim C_{n_{k}}=\lim \inf C_{n^{\circ}}$ The sequence $\left\{g_{n}^{\prime}\right\}$ converges uniformly to $g^{\prime}$ on compact subsets of $D$; thus, for each $z \in D$,

$$
\left(1-|z|^{2}\right) g^{*}(z)=\lim \left(1-|z|^{2}\right) g_{n_{k}}^{*}(z) \leq \lim C_{n_{k}}
$$

Thus

$$
C_{8} \leq \lim \inf C_{n^{*}}
$$

If (2.11) holds for some $r(0 \leq r<1)$, then

$$
\begin{aligned}
\lim \sup C_{n} & =\lim \sup \left(\max _{|z| \leq r}\left(1-|z|^{2}\right) g_{n}^{*}(z)\right) \\
& =\max _{|z| \leq r}\left(1-|z|^{2}\right) g^{*}(z) \leq C_{g^{\circ}}
\end{aligned}
$$

By (2.10) and (2.12), we have $C_{g}=\lim C_{n}$.

Returning to the sequence $f_{n}(z)=M z^{-n}(n=2,3, \ldots), C_{n}=C_{f_{n}}$, set

$$
g_{n}(w)=f_{n}\left(\frac{w-x_{n}}{1-x_{n} w}\right) \text {, }
$$

where $x_{n}$ is determined from an arbitrary fixed number $b>0$ by $x_{n}=e^{-b / n}$. Then $C_{n}=C_{8_{n}}$ by (1.2). From (2.8), if $|z|=\left(n-t_{n}\right) /(n+1)$, then $C_{n}=\left(1-|z|^{2}\right) f_{n}^{*}(z)$. We choose $z_{n}=-\left(n-t_{n}\right) /(n+1)$. Then $C_{n}=\left(1-\left|w_{n}\right|^{2}\right) g_{n}^{*}\left(w_{n}\right)$, where $w_{n}$ is determined by $\left(w_{n}-x_{n}\right) /\left(1-x_{n} w_{n}\right)=z_{n}$. We find

$$
w_{n}=\frac{1+t_{n}-b+o(1)}{1+t_{n}+b+o(1)} \text {. }
$$

Since $0<t_{n}<M^{-2}$, the condition (2.11) in Lemma 1 holds for all sufficiently large values of $n$. Therefore,

$$
\lim C_{n}=C_{8}
$$

where $g$ is the limit of the sequence $\left\{g_{n}\right\}$. A direct calculation yields

$$
g(w)=\lim M\left(\frac{1-e^{-b / n} w}{w-e^{-b / n}}\right)=M \exp \left(b \frac{1+w}{1-w}\right) .
$$

As noted in (2.3), $C_{8}=C_{M}^{*}$. This reestablishes Example 1 .

3. Estimates on Koebe sequences. We now obtain some estimates for the growth of the modulus of a normal function on a Koebe sequence of arcs. 
Theorem 1. Let $f$ be meromorpbic in $D$ with order of normality $C(0<C \leq+\infty)$. Let $\gamma$ be an open subarc of $\Gamma$ and let $\left\{\gamma_{n}\right\}$ be a Koebe sequence of arcs relative to $\gamma$. Set $M=\sup _{\zeta \epsilon \gamma}|f(\zeta)|$. If $f$ is not bounded on $\gamma$, then

$$
\lim \inf M_{n} \geq \frac{1+\left(1+C^{2}\right)^{1 / 2}}{C} \exp \left[-\left(1+C^{2}\right)^{1 / 2}\right]
$$

and if $f$ is bounded on $y_{0}$ then

$$
\lim \inf M_{n} \geq M
$$

Also, if $0<C<+\infty$ and $f$ is analytic in a domain $G \subset D$ for which $\gamma$ is a free boundary arc, then

$$
\lim \inf M_{n} \geq M
$$

Proof. If $C=+\infty$, we let the right-hand side of (3.1) equal zero. Thus we assume that $0<C<+\infty$. If $f$ is not bounded on $\gamma$, we can choose a point $\zeta^{*} \in \gamma$ such that

$$
\left|f\left(\zeta^{*}\right)\right|>\frac{1+\left(1+C^{2}\right)^{1 / 2}}{C}
$$

Let $\gamma^{*}$ be an open arc such that $\zeta^{*} \in \gamma^{*}$ and the closure of $\gamma^{*}$ lies in $\gamma$. Let $\zeta$ and $\zeta^{\prime}$ denote the left and right endpoints, respectively, of $\gamma^{*}$ as viewed from $z=0$, and let $r$ and $r^{\prime}$ be the radii at $\zeta$ and $\zeta$, respectively. For all sufficiently large values of $n$, the arc $\gamma_{n}$ has a Jordan subarc $\gamma_{n}^{*}$ with an endpoint on each of $r$ and $r^{\prime}$ and otherwise lying in the sector subtending $\gamma^{*}$. Let $r_{n}$ and $r_{n}^{\prime}$ denote the segments on $r$ and $r^{\prime}$, respectively, such that the arc

$$
\Gamma_{n}=r_{n} \cup \gamma_{n}^{*} \cup r_{n}^{\prime}
$$

is a Jordan crosscut of $D$. The region bounded by $\Gamma_{n} \cup \Gamma-\gamma^{*}$ is denoted by $D_{n}$. Then $\lim D_{n}=D$ in the obvious sense. For all sufficiently large values of $n$, we can choose a point $z_{n} \in D_{n}$ such that

$$
\left|f\left(z_{n}\right)\right|>\frac{1+\left(1+C^{2}\right)^{1 / 2}}{C}
$$

and $\lim z_{n}=\zeta^{*}$. By the principle of monotoneity for harmonic measures,

$$
\omega\left(z_{n}, \gamma^{*}, D\right) \leq \omega\left(z_{n}, \Gamma_{n}, D_{n}\right)
$$

Let $z=\phi_{n}(w), z_{n}=\phi_{n}(0)$, be a conformal map of $|w|<1$ onto $D_{n}$. Then $\Gamma_{n}=$ $r_{n} \cup \gamma_{n}^{*} \cup r_{n}^{\prime}$ corresponds to a subarc $\Lambda_{n} \cup \Lambda_{n}^{*} \cup \Lambda_{n}^{\prime}$ of $|w|=1$ subtended by a central angle of measure $\left(\delta_{n}+\delta_{n}^{*}+\delta_{n}^{\prime}\right) / 2 \pi$, where $r_{n}$ corresponds to the subarc $\Lambda_{n}$ subtended by a central angle of measure $\delta_{n} / 2 \pi$, etc. Since $\left\{z_{n}\right\}$ converges to $\zeta^{*}, \lim \omega\left(z_{n}, \gamma^{*}, D\right)=1$; thus, by (3.4) and the invariance of harmonic measure under $\phi_{n}$, 


$$
\lim \left(\delta_{n}+\delta_{n}^{*}+\delta_{n}^{\prime}\right) / 2 \pi=1
$$

By performing rotations if necessary, we can assume that the arcs $\Lambda_{n}$ have a common right endpoint as viewed from $w=0$. The segments $r_{n}$ converge uniformly to $\zeta$; thus, the sequence $\left\{\phi_{n}\right\}$ converges uniformly to $\zeta$ on $\cap \Lambda_{n}$. If $\cap \Lambda_{n}$ is a proper subarc of $|w|=1$, it follows that $\left\{\phi_{n}\right\}$ converges uniformly to $\zeta$ on compact subsets of $|w|<1$ (see [4, Corollary 1] or [6, Theorem 10]). However, $\phi_{n}(0)=z_{n}$ and $\lim z_{n}=\zeta^{*} \neq \zeta$. Thus $\bigcap \Lambda_{n}$ is a singleton and $\lim \delta_{n}=0$. In a similar fashion it is seen that $\lim \delta_{n}^{\prime}=0$. From (3.5),

$$
\lim \delta_{n}^{*}=2 \pi
$$

Let $G_{n}$ be the lens bounded by $\Lambda_{n}^{*}$ and the circle through $w=0$ joining the endpoints of $\Lambda_{n}^{*}$. Since $\Lambda_{n}^{*}$ is subtended by the central angle $\delta_{n}^{*}$, it follows from some simple geometric considerations that $G_{n}$ has vertex angle $a_{n}$ given by

$$
a_{n}=\pi-1 / 2 \delta_{n}^{*}
$$

We now apply the estimate of Theorem $A$ to the function $f\left(\phi_{n}(w)\right)$. First, set

$$
\begin{aligned}
& M_{n}^{*}=\sup _{w \in \Lambda_{n}^{*}}\left|f\left(\phi_{n}(w)\right)\right| \leq M_{n}, \\
& \lambda_{n}=\lambda_{a_{n}}=\frac{a_{n}}{2 \sin a_{n}}, \\
& \mu_{n}=\mu_{a_{n}}=\frac{1+\left(1+4 C^{2} \lambda_{n}^{2}\right)^{1 / 2}}{2 C \lambda_{n}} .
\end{aligned}
$$

Since

$$
\left|f\left(\phi_{n}(0)\right)\right|=\left|f\left(z_{n}\right)\right|>\frac{1+\left(1+C^{2}\right)^{1 / 2}}{C}>\mu_{n},
$$

we use $\mu=\mu_{n}$ in (2.1) to obtain the estimate

$$
M_{n} \geq M_{n}^{*} \geq \mu_{n} \exp \left[-C \lambda_{n}\left(\mu_{n}+1 / \mu_{n}\right)\right]
$$

By (3.6) and (3.7), $\lim \lambda_{n}=1 / 2$; thus,

$$
\lim \mu_{n}=\frac{1+\left(1+C^{2}\right)^{1 / 2}}{C}
$$

The estimate (3.1) now follows.

Before we continue with the proof of Theorem 1, we cite Bagemihl and Seidel's result $[1$, Theorem 1$]$ which shall be used later in our proof.

Corollary 1. Let $f$ be meromorphic in $D$ with order of normality $C(0<C<$ $+\infty)$. Then $f$ bas no Koebe values. 
Proof. Let $\gamma$ be any open subarc of $\Gamma$. If $f$ is bounded on $\gamma$, then Koebe's lemma implies that $w=0$ cannot be a Koebe value of $f$ on a Koebe sequence of arcs relative to $\gamma$. If $f$ is not bounded on $\gamma$, then the estimate (3.1) implies the same conclusion. If $w=c$ is a Koebe value of $f$, then we consider $F(z)=f(z)-c$ or $F(z)=1 / f(z)$, depending on whether $c$ is finite or $c=\infty$. In either case, $F$ is normal and therefore cannot have zero as a Koebe value; thus $c$ cannot be a Koebe value of $f$ and the corollary is proved.

We now retum to the proof of Theorem 1. If $f$ is bounded on $\gamma$, then, by Fatou's theorem, $f$ has radial limits almost everywhere on $\gamma$. The estimate (3.2) now follows from the maximum principle as noted by MacLane [7, Theorem 9]. (Let $\gamma^{*}$ be any closed subarc of $\gamma$ such that $f$ has a radial limit at each endpoint of $\gamma^{*}$. The maximum principle is applied to $f$ in the region bounded by $\gamma_{n}^{*}, r^{\prime}, \gamma_{n+1}^{*}, r$; our notation here is that used in the proof of (3.1). It is worth noting that if $M$ is the maximum of $|f(\zeta)|$ for $\zeta$ in the closure of $\gamma$, then equality holds in (3.2).)

Now suppose $f$ is analytic in a domain $G \subset D$ for which $\gamma$ is a free boundary arc. If $M$ is finite, then we just proved the desired estimate (3.3) (even if $C=+\infty$ ). Therefore assume $M=+\infty$ and $0<C<+\infty$. Then either (i) there is a point $\zeta^{*} \in \gamma$ at which $f$ is unbounded or (ii) for each open subarc $\gamma^{*}$ of $\gamma$,

$$
M^{*}=\sup _{\zeta \in \gamma^{*}}|f(\zeta)|<+\infty \text {, }
$$

and sup $M^{*}=+\infty$. If (ii) occurs, then (3.2) implies that for each open subarc $\gamma^{*} \subset \gamma$,

$$
\lim \inf M_{n} \geq M^{*} \text {. }
$$

Thus $\lim M_{n}=+\infty=M$.

Finally, suppose (i) occurs. (It can be deduced from a theorem of McMillan $[8$, Theorem 1] that if

$$
\lim \sup M_{n_{k}}<+\infty
$$

for any subsequence $\left\{\gamma_{n_{k}}\right\}$, then $\infty$ is a Koebe value of $f$ on a Koebe sequence of arcs relative to a subarc of $\gamma$ having $\zeta^{*}$ as one endpoint. Since $0<C<+\infty$, $f$ has no Koebe values; it follows that $\lim M_{n}=+\infty$ as required. We sketch a proof that in spirt is somewhat similar to that of McMillan. It is also similar to the type of argument used by Barth and Schneider [2] to prove MacLane's lemma.) Let us first assume that ${ }^{\prime}{ }^{\prime}$ can be chosen so that

$$
M_{n}<M^{\prime} \quad(n=1,2, \ldots) .
$$

Select an open subarc $\gamma^{*}$ for which the closure lies in $\gamma$ and such that $\zeta^{*} \epsilon \gamma^{*}$. Let $\Delta$ be the open sector subtending $\gamma^{*}$. Since $\gamma$ is a free boundary arc of $G$, there exists an $\epsilon>0$ such that $G$ contains the set 


$$
\Delta_{\epsilon}=\Delta \cap\{z: 1-\epsilon<|z|<1\} .
$$

Choose a sequence $\left\{z_{k}\right\}$ in $\Delta_{\epsilon}$ such that $\lim z_{k}=\zeta^{*}$ and such that

$$
\left|f\left(z_{k}\right)\right|>M^{\prime}+k \quad(k=1,2, \ldots) .
$$

We can also assume that the Riemann surface of $f$ over the extended plane (sphere) has no branch points over any of the radial segments

$$
R_{k}=\left\{w:\left|w_{k}\right| \leq|w|<+\infty \text {, arg } w=\arg w_{k}, w_{k}=f\left(z_{k}\right)\right\} \text {. }
$$

If the regular element $e_{z_{k}}\left(w, w_{k}\right)$ of $z=f^{-1}(w)$ is continued along $R_{k}$, there are two possibilities: either the continuation defines a transcendental singularity in a finite distance or at $w=\infty$, or the continuation defines a regular or algebraic element at $w=\infty$. In each case, the branch of $f^{-1}$ defined by the continuation maps the segment of $R_{k}$ in question onto a Jordan arc $\gamma_{k}^{\prime}$ with initial point $z_{k}$. By (3.8) and (3.9), $\gamma_{k}^{\prime} \cap \gamma_{n}=\varnothing(n=1,2, \ldots)$. In the first case, $\gamma_{k}^{\prime}$ terminates at a point of $\Gamma-\gamma ;$ in the second case, $\gamma_{k}^{\prime}$ terminates at a pole of $f$ outside $\Delta_{\epsilon}$. It is apparent that from the sequence $\left\{\gamma_{k}^{\prime}\right\}$ we can find a Koebe sequence of arcs on which $f$ has Koebe value $\infty$. This contradiction shows that the condition (3.8) cannot hold; that is, $\lim \sup M_{n}=+\infty$. By the same argument, $\lim \sup M_{n_{k}}=+\infty$ for any subsequence $\left\{\gamma_{n_{k}}\right\}$. Thus, $\lim M_{n}=+\infty$, and this completes the proof of (i).

We shall make use of the estimate (3.3) in the following form.

Corollary 2 Let $f$ be meromorphic and normal in $D$. Let $\gamma$ be a subarc of $\Gamma$ sucb that $f$ is analytic in a domain $G C D$ and $\gamma$ is a free boundary arc of $G$. If $f$ is bounded on a Koebe sequence of arcs relative to $\gamma$, then $f$ is bounded on $\gamma$; moreover,

$$
M=\sup _{\zeta \in \gamma}|f(\zeta)| \leq \lim \inf M_{n}<+\infty
$$

Since $f$ and $1 / f$ have the same order of normality, we can apply Theorem 1 to $1 / f$ to obtain the following result.

Corollary 3. Let $f$ be meromorphic in $D$ with order of normality $C(0<C \leq$ $+\infty)$. Let $\gamma$ be an open subarc of $\Gamma$ and let $\left\{\gamma_{n}\right\}$ be a Koebe sequence of arcs relative to $\gamma_{0}$ Set

$$
m=\inf _{\zeta \in \gamma}\left(\liminf _{z \rightarrow \zeta}|f(z)|\right)
$$

If $w=0$ is a boundary value of $f$ on $\gamma$, then

$$
\lim \sup m_{n} \leq \frac{C}{1+\left(1+C^{2}\right)^{1 / 2}} \exp \left[\left(1+C^{2}\right)^{1 / 2}\right] ;
$$

and if $w=0$ is not a boundary value of $f$ on $\gamma$, then

$$
\lim \sup m_{n} \leq m_{\text {. }}
$$


Also, if $0<C<+\infty$ and $f$ omits $w=0$ is a domain $G \subset D$ for which $\gamma$ is a free boundary arc, then

$$
\lim \sup m_{n} \leq m
$$

The following example is concemed with the sharpness of the estimate (3.1) of Theorem 1 .

Example 2. Let $w=\phi(z)$ be an analytic map of $D$ into $|w|<1$ such that $w=0$ is a boundary value of $\phi$ at $\zeta=1$ and

$$
\lim _{|z| \rightarrow 1 ; z \in \sigma}|\phi(z)|=1
$$

where $\sigma$ is a spiral in $D$ (see $\left[11\right.$, p. 14]). Let $g_{n}(w)=M w^{-n}$ and set $f_{n}(z)=$ $\boldsymbol{g}_{n}(\phi(z))$. By (1.2) and Example 1,

$$
C_{f_{n}} \leq C_{g_{n}}=C_{M}^{*}+o(1)
$$

Also, $f_{n}$ is unbounded at $\zeta=1$ so that the estimate (3.1) applies. Since

$$
\lim _{|z| \rightarrow 1 ; z \in \sigma}\left|f_{n}(z)\right|=M,
$$

(3.1) can be written as $C_{M}^{*} \leq C_{f_{n}}$. Thus

$$
C_{M}^{*} \leq C_{f_{n}} \leq C_{M}^{*}+o(1)
$$

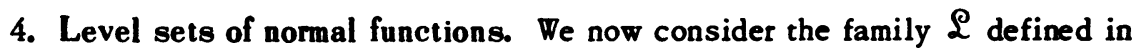
the introduction.

Lemma 2. Suppose the nonconstant normal meromorphic function $f \notin \mathcal{L}$. Then (i)

$$
C^{*} \leq C=C_{f}
$$

If the level set $L_{R}$ of $f$ contains a Koebe sequence of arcs $\left\{\gamma_{n}\right\}$ relative to the open subarc $\gamma$ of $\Gamma$, then the following bold:

$$
\frac{1+\left(1+C^{2}\right)^{1 / 2}}{C} \exp \left[-\left(1+C^{2}\right)^{1 / 2}\right] \leq R \leq \frac{C}{1+\left(1+C^{2}\right)^{1 / 2}} \exp \left[\left(1+C^{2}\right)^{1 / 2}\right]
$$

(iii) if $\zeta$ is any point of $\gamma$ and

$$
R^{\prime}<\frac{1+\left(1+C^{2}\right)^{1 / 2}}{C} \exp \left[-\left(1+C^{2}\right)^{1 / 2}\right]
$$

then, in each neighborbood of $\zeta$, there is a component $U$ of the set $f^{-1}\left(|w|<R^{\prime}\right)$ and a component $V$ of the set $f^{-1}\left(|w|>1 / R^{\prime}\right)$ such that the closures of $U$ and $V$ lie in $D$.

Proof. We first verify (ii). Both $f$ and $1 / f$ must be unbounded on $\gamma$ (and in fact on each subarc of $\gamma$ ), otherwise the assumption concerning $L_{R}$ is 
impossible by MacLane's lemma. Since $R=\lim M_{n}=\lim m_{n}$, the inequality (ii) follows from (3.1) and (3.10).

If $f \notin \mathcal{L}$, then there exists an $R$ such that (ii) holds for $C=C_{f}$; thus $C^{*} \leq C$.

Finally, we verify (iii). Let $\zeta \in \gamma$. Since $f$ and $1 / f$ are unbounded on each subarc of $\gamma$, Corollary 2 implies that $\zeta$ is a limit point of poles and zeros of $f$. For each positive integer $k$, let $U_{k}$ be a component of $f^{-1}\left(|w|<R^{\prime}\right)$ containing a zero $z_{k}$ of $f$, and let $V_{k}$ be a component of $f^{-1}\left(|w|>1 / R^{\prime}\right)$ containing a pole $p_{k}$ of $f$, where $z_{k} \rightarrow \zeta$ and $p_{k} \rightarrow \zeta$. By (ii), the level sets $L_{R^{\prime}}$ and $L_{1 / R^{\prime}}$ cannot contain a Koebe sequence of arcs. Thus the diameters of $U_{k}$ and $V_{k}$ tend to zero as $k \rightarrow+\infty$. Because $L_{R}$ contains the Koebe sequence $\left\{\gamma_{n}\right\}$, the closures of $U_{k}$ and $V_{k}$ lie in $D$ for all sufficiently large values of $k$.

The next result follows from (i) and (iii) of Lemma 2.

Theorem 2. Let $f$ be meromorphic in $D$ with order of normality $C(0<C<$ $+\infty)$. If either $f$ is analytic in $D$ or $C<C^{*}$, then $f \in \mathcal{L}$.

We now show that $C^{*}$ cannot be replaced by any larger number in Lemma 2 and Theorem 2.

Theorem 3. $C^{*}=\inf \left\{C_{f}: f \notin \mathscr{L}, C_{f}>0\right\}$.

Proof. Lemma 2 states that $C^{*}$ is a lower bound for the set in question. For each value $k=3,4, \cdots$, let $T_{k}$ be a regular non-Euclidean polygon in $D$ with $k$ sides and center $z=0$ and such that each vertex angle of $T_{k}$ has magnitude $2 \pi / 3$. Let $n$ be a fixed positive integer, and let $w=f_{k}(z)$ be an analytic $n$-to-1 map of $T_{k}$ onto $|w|<1$ such that $f_{k}(z)=0$ only for $z=0$. We extend $f_{k}$ from $T_{k}$ to all of the unit disk $D$ by the usual reflection technique. It is apparent that the level set $L_{1}$ of $f_{k}$ consists of the boundary of $T_{k}$ and all its reflections. Thus $L_{1}$ contains a Koebe sequence of arcs relative to $\Gamma$ and hence $f_{k} \notin £$.

We intend to show that $f_{k}$ can be constructed so that

$$
\lim _{k \rightarrow+\infty} f_{k}(z)=z^{n}
$$

uniformly on compact subsets of $D$ and

$$
C_{f_{k}}=\left(1-\left|z_{k}\right|^{2}\right) f_{k}^{*}\left(z_{k}\right)
$$

where $\left|z_{k}\right| \leq r<1$. Then, by Lemma 1 ,

$$
\lim C_{f_{k}}=C_{n},
$$

where $C_{n}$ is the order of normality of $z^{n}$. By Example 1,

$$
\lim C_{n}=C^{*} \text {. }
$$


Since $f_{k} \notin \mathscr{L}$, this implies the assertion of our theorem.

Because the expression $\left(1-|z|^{2}\right) f_{k}^{*}(z)$ is invariant under reflections through non-Euclidean lines of the unit disk $D$,

$$
C_{f_{k}}=\sup _{z \in T_{k}}\left(1-|z|^{2}\right) f_{k}^{*}(z)
$$

Also $D$ is the kernel of the domains $T_{k}$. Thus, we need only concern ourselves with the construction of $f_{k}$ on $T_{k}$. Let $T_{k}$ be positioned so that there is a vertex of $T_{k}$ located on the positive real axis at $r_{k}$. The conformal map $z=b_{k}(w)$ of $|w|<1$ onto $T_{k}$ such that $b_{k}(0)=0$ and $b_{k}(1)=r_{k}$ can be given in terms of hypergeometric functions as follows (see $[5$, p. 83]):

$$
z=b_{k}(w)=r_{k} s_{k} \psi_{2}(w) / \psi_{1}(w),
$$

where

$$
\psi_{1}(w)=F\left(\frac{1}{6}-\frac{1}{k}, \frac{1}{6}, 1-\frac{1}{k} ; w^{k}\right), \quad \psi_{2}(w)=w F\left(\frac{1}{6}+\frac{1}{k}, \frac{1}{6}, 1+\frac{1}{k} ; w^{k}\right),
$$

and

$$
s_{k}=\frac{\psi_{1}(1)}{\psi_{2}(1)}=\frac{\Gamma(1-1 / k) \Gamma(5 / 6+1 / k)}{\Gamma(1+1 / k) \Gamma(5 / 6-1 / k)} .
$$

Let $w=g_{k}(z)$ be the inverse of $b_{k}$ and set $w=f_{k}(z)=\left[g_{k}(z)\right]^{n}$. We have $\lim b_{k}(w)=w$ uniformly on $|w| \leq 1$. Thus,

$$
\lim f_{k}(z)=z^{n}
$$

uniformly on compact subsets of $D$. By Lemma $1, C_{n}$, the order of normality of $z^{n}$, satisfies

$$
0<C_{n} \leq \lim \inf C_{f_{k}}
$$

We now show that $f_{k}^{\prime}$, and hence $f_{k}^{*}$, is bounded independent of $k$ on the closure of $T_{k}$. First,

$$
\begin{aligned}
b_{k}^{\prime}(w) & =r_{k} s_{k} \frac{\psi_{1} \psi_{2}^{\prime}-\psi_{2} \psi_{1}^{\prime}}{\psi_{1}^{2}} k w^{k-1} \\
& =r_{k} s_{k} \frac{1 / k}{\left(w^{k}\right)^{1+1 / k}\left(1-w^{k}\right)^{1 / 3} \psi_{1}^{2}} k w^{k-1}=r_{k} s_{k} \frac{1}{\left(1-w^{k}\right)^{1 / 3} \psi_{1}^{2}} .
\end{aligned}
$$

The quantity $\psi_{1} \psi_{2}^{\prime}-\psi_{2} \psi_{1}^{\prime}$ has the above simplification using the technique of Carathéodory [3, p. 164]. Thus,

$$
\left|h_{k}^{\prime}(w)\right| \geq \frac{r_{3} s_{3}}{2^{1 / 3}} \frac{1}{\left|\psi_{1}\right|^{2}} \geq \frac{r_{3} s_{3}}{2^{1 / 3}} \frac{1}{F^{2}(1 / 6,1 / 6,2 / 3 ; 1)},
$$


and

$$
\left|f_{k}^{\prime}(z)\right| \leq n\left|g_{k}^{\prime}(z)\right| \leq n \frac{2^{1 / 3}}{r_{3} s_{3}} F^{2}\left(\frac{1}{6}, \frac{1}{6}, \frac{2}{3} ; 1\right) .
$$

It now follows that there exists a compact set $|z| \leq r<1$ such that $C_{f_{k}}=$ $\left(1-\left|z_{k}\right|^{2}\right) f_{k}^{*}\left(z_{k}\right)$, where $\left|z_{k}\right| \leq r$; for if $\left|z_{k}\right| \rightarrow 1$, then (since $f_{k}^{*}$ is bounded independent of $k$ on the closure of $T_{k}$ ) $\lim C_{f_{k}}=0$. This contradicts (4.1).

5. Functions bounded on Koebe sequences. Although a nonconstant normal meromorphic function $f$ cannot have a Koebe value, $|f|$ can have a Koebe value $\boldsymbol{R}$ as evidenced by the functions of Examples 1 and 2 and Theorem 3. It must be the case that $0<R<+\infty$. Also, a function $f$ can be "very normal" and yet $|f|$ can have a Koebe value. For example, if $M>0$ and $f(z)=z / M$ then $|f|$ has a Koebe value and $C_{f}=1 / M$.

Let $f$ be meromorphic and normal in $D$. Suppose $|f|$ has $R$ as a Koebe value on a Koebe sequence of arcs $\left\{\gamma_{n}\right\}$ relative to an open subarc $\gamma$ of $\Gamma$. If no point of $\gamma$ is a limit of poles of $f$, then Corollary 2 yields $|f(\zeta)| \leq R(\zeta \in \gamma)$. By Fatou's theorem, $f$ has radial limits at almost every point of $\gamma$; these radial limits all have modulus $R$. If no point of $\gamma$ is a limit point of zeros of $f$, then Corollary 2 applied to $1 / f$ yields

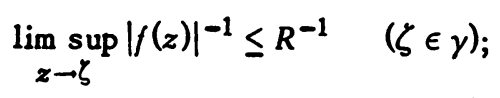

that is, $f$ has no boundary values $w$ on $\gamma$ such that $|w|<R$. On the other hand suppose $\zeta \epsilon \gamma$ is a limit point of zeros of $f$; let $z_{k} \rightarrow \zeta$ be such a sequence of zeros. Choose $R^{\prime}\left(0<R^{\prime}<R\right)$ such that the Riemann surface of $f$ has no branch points over the circle $|w|=R^{\prime}$. Let $U_{k}$ be the component of $f^{-1}\left(|w|<R^{\prime}\right)$ containing $z_{k}$. For all sufficiently large values of $k, U_{k}$ cannot intersect any of the arcs $\gamma_{n}$ since

$$
\lim m_{n}\left(\gamma_{n} ; f\right)=R>R^{\prime} \cdot \text {. }
$$

Since $f$ has radial limits of modulus $R>R^{\prime}$ at almost every point of $\gamma_{\text {, we con- }}$ clude that the diameter of $U_{k}$ tends to zero as $k \rightarrow+\infty$. Thus $|w| \leq R^{\prime}$ lies in the set of boundary values of $f$ at $\zeta$. Since $R^{\prime}$ can be chosen arbitrarily close to $R$, we conclude that $|w| \leq R$ is the set of boundary values of $f$ at $\zeta$.

In the foregoing discussion, we have assumed that no point of $\gamma$ is a limit point of poles of $f$. The following theorem shows that this is the case if $C_{f}$ is sufficiently small.

Theorem 4. Let / be meromorpbic in $D$ with order of normality $C>0$. Let $|f|$ bave the Koebe value $R$ on a Koebe sequence of arcs $\left\{\gamma_{n}\right\}$ relative to an open subarc $\gamma$ of $\Gamma$. If $C<C^{*}$, then eitber $f$ or $1 / f$ is bounded on $\gamma$. 
Proof. Suppose both $f$ and $1 / f$ are unbounded on $\gamma$. Then, by (3.1),

and by (3.10),

$$
R=\lim M_{n} \geq \frac{1+\left(1+C^{2}\right)^{1 / 2}}{C} \exp \left[-\left(1+C^{2}\right)^{1 / 2}\right]
$$

$$
R=\lim m_{n} \leq \frac{C}{1+\left(1+C^{2}\right)^{1 / 2}} \exp \left[\left(1+C^{2}\right)^{1 / 2}\right]
$$

Because $C<C^{*}$, these inequalities are inconsistent. Thus, either $f$ or $1 / f$ is bounded on $\gamma$.

\section{REFERENCES}

1. F. Bagemihl and W. Seidel, Koebe arcs and Fatou points of normal functions, Comment. Math. Helv. 36 (1961), 9-18. MR 25 \#5183.

2. K. F. Barth and W. J. Schneider, A short proof of a lemma of G. R. MacLane, Proc. Amer. Math. Soc. 20 (1969), 604-605. MR 38 \#4688.

3. C. Carathéodory, Funktionentheorie, Band 2, Birkhäuser, Basel, 1950; English transl., Theory of functions of a complex variable. Vol. 2, Chel sea, New York, 1954. MR 12, 248; 16, 346.

4. S. Dragosh, Sequences of normal meromorphic functions, Arch. Math. 23 (1972), 183-187.

5. G. M. Goluzin, Geometric theory of functions of a complex variable, GITTL, Moscow, 1952; English transl., Transl. Math. Monograph s, vol. 26, Amer. Math. Soc., Providence, R. I., 1969. MR 15; 112; 40 \#308.

6. O. Lehto and K. I. Virtanen, Boundary behaviour and normal meromorphic functions, Acta Math. 97 (1957), 47-65. MR 19, 403.

7. G. R. MacLane, Asymptotic values of holomorphic functions, Rice Univ. Studies, 49, no. 1 (1963), 83 pp. MR $26 \# 6419$.

8. J. E. McMillan, $O_{n}$ local asymptotic properties, the asymptotic value sets, and ambiguous properties of functions meromorphic in the open unit disc, Ann. Acad. Sci. Fenn. Ser. AI No. 384 (1965), 1-12. MR 35 \#4431.

9. R. Nevanlinna, Eindeutige analytische Funktionen, 2nd ed., Springer-Verlag, Berlin, 1953; English transl., Die Grundlehren der math. Wissenschaften, Band 162, Springer-Verlag, Berlin and New York, 1970. MR 15, 208; 43 \#5003.

10. K. Noshiro, Contributions to the theory of meromorphic functions in the unit circle, J. Fac. Sci. Hokkaido Imperial Univ. 7 (1939), 149-159.

11. W. Seidel, On the cluster values of analytic functions, Trans. Amer. Math. Soc. 34 (1932), 1-21.

DEPARTMENT OF MATHEMATICS, MICHIGAN STATE UNIVERSITY, EAST LANSING, MICHIGAN 48823 